\title{
Rechallenge with temozolomide with different scheduling is effective in recurrent malignant gliomas
}

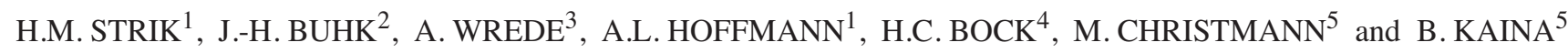 \\ Departments of ${ }^{1}$ Neurology, ${ }^{2}$ Neuroradiology, ${ }^{3}$ Neuropathology and ${ }^{4}$ Neurosurgery, University of Göttingen, \\ Göttingen; ${ }^{5}$ Department of Toxicology, University of Mainz, Mainz, Germany
}

Received July 8, 2008; Accepted September 17, 2008

DOI: $10.3892 / \mathrm{mmr}+00000042$

\begin{abstract}
Treatment of recurrent malignant glioma, which has a poor patient prognosis, has not been standardised. Moreover, it is unclear whether repeated treatment with temozolomide is effective in patients who received previous temozolomide treatment before developing a recurrence. Here, we present the results of a high-dose individually adapted 21-day regimen demonstrating that rechallenge is effective even in patients expressing $\mathrm{O}^{6}$-methylguanine-DNA methyltransferase (MGMT) in the tumor. Twenty-one patients with recurrent malignant gliomas pre-treated with temozolomide, 18 WHO IV glioblastoma (GBM) and 3 WHO III patients, received $100 \mathrm{mg} / \mathrm{m}^{2}$ temozolomide on days $1-21 / 28$. The GBM patients had a median Karnofsky performance status of $60 \%$ and a median age of 54.8 years. Blood counts decreased continuously, enabling a gradual dose adaptation. When blood counts dropped below normal values, temozolomide was applied on days $1-5 / 7$. Dosage was reduced to $50-75 \mathrm{mg} / \mathrm{m}^{2}$ in 11 patients and gradually increased up to $130 \mathrm{mg} / \mathrm{m}^{2}$ in 3 patients. WHO grade $3 / 4$ toxicity was hematological in 3 patients and non-hematological in 3 patients. In GBM patients $(n=18)$, response after $>3$ months was complete in 3 patients, partial in $1(22 \%)$, stable disease in $7(39 \%)$ and progressive disease in $7(39 \%)$. Progression-free survival at 6 months (PFS-6M) was 39\%. Median survival was 9.1 months from relapse and 17.9 months overall. Of the patients with unmethylated MGMT promoter, 2/7 were progression-free for $>6$ (15 and 19) months. The data indicate that rechallenge with near-continuous, higher-dose temozolomide $\left(100 \mathrm{mg} / \mathrm{m}^{2}\right.$ on days $1-21 / 28$ or days $1-5 / 7$ with individual dose adaptation) is also feasible in patients with critical blood counts. Objective responses can be achieved even after relapse during a conventional 5/28-day regimen. The resistance of tumors
\end{abstract}

Correspondence to: Dr Herwig Strik, Department of Neurology, University of Göttingen, Robert Koch Strasse 40, D-37099 Göttingen, Germany

E-mail: herwig.strik@med.uni-goettingen.de

Key words: glioblastoma, glioma, chemotherapy, temozolomide, $\mathrm{O}^{6}$-methylguanine-DNA methyltransferase characterized by unmethylated MGMT promoter may be overcome by near continuous temozolomide administration, which is probably most effective with a 5/7-day schedule. In spite of the relatively poor clinical prognosis, the data indicate that rechallenge with temozolomide with a dose-dense and long-lasting administration protocol is tolerable and comparable with other reported treatment protocols involving temozolomide.

\section{Introduction}

Despite considerable efforts in the last decades and remarkable progress in neurosurgery, radiotherapy and the development of new chemotherapeutic drugs, malignant gliomas are still among the most difficult human cancers to treat. Overall survival ranges from 12 to 17 months with combined modality treatment $(1,2)$. Relapse is difficult to control, with a response rate of $9 \%$ with different salvage strategies, a median survival of 30 weeks after relapse and a 6-month progression-free survival (PFS-6M) of $15 \%$ (3). With nitrosourea-based secondline chemotherapy, a PFS-6M of approximately $40 \%$ and median survival of up to 38 weeks can be achieved, while the rate of objective response remains at $9-15 \%$ (4). A conventional $5 / 28$-day regimen of temozolomide can achieve response rates of approximately 5\% and a PFS-6M of $21 \%$ (5). Regardless, temozolomide has become a standard treatment in the primary therapy of glioblastomas (GBMs) (2). However, it is unclear whether re-exposure to the same regimen can result in tumor control, especially if the recurrence occurs during primary chemotherapy.

$\mathrm{O}^{6}$-methylguanine-DNA methyltransferase (MGMT), a suicide enzyme reverting the cytotoxic effect of nitrosureas or temozolomide, has proven to be a major predictor of response to chemotherapy in GBMs (6). Importantly, MGMT is the first prognostic factor in gliomas that can be influenced by therapy and can, for example, be depleted by prolonged application of temozolomide (7). Moreover, prolonged schedules allow for an increase in temozolomide of up to double the conventional dose per cycle. This suggests the possibility of re-exposing recurrent malignant gliomas to temozolomide in dose-dense schedules. Here, we analyzed our results regarding the efficacy of temozolomide treatment on days 1-21 of 28 at an increased dose of $100 \mathrm{mg} / \mathrm{m}^{2}$, as compared with the commonly used dose of $75 \mathrm{mg} / \mathrm{m}^{2}$, in recurrent malignant gliomas (8). 


\section{Patients and methods}

From May 2005, twenty-one consecutive patients in stable clinical condition were treated at the Department of Neurology, University of Göttingen with oral temozolomide $100 \mathrm{mg} / \mathrm{m}^{2}$ on days $1-21 / 28$. Recurrence or progression was diagnosed by MRI according to the MacDonald criteria (progression, $>25 \%$ increase in contrast-enhancing lesions) (9). Perfusion- and diffusion-weighted MRI was used to obtain additional information on tumor biology. Treatment was administered with the informed consent of the patients.

Patients with a total leukocyte count $>4,000 / \mu 1$, thrombocytes $>100,000 / \mu 1$ and a Karnofsky performance status (KPS) of $\geq 50 \%$ were treated. Those that completed at least 1 course of treatment are reported.

To enable good comparability with the literature, treatment results are reported for GBM patients. Data on toxicity are reported for all patients.

Glioblastomas. The main characteristics of the GBM patients are reported in Table I. The histopathological records of all patients and histological slides of all but 1 patient were reviewed at our Department of Neuropathology, and the diagnosis of GBM confirmed. Local BCNU was administered to 2 patients at primary surgery.

All patients were pre-treated with temozolomide after initial diagnosis. After primary surgery, all but 1 patient received $75 \mathrm{mg} / \mathrm{m}^{2}$ concomitant temozolomide daily with focal involved-field irradiation. All patients received at least one cycle of $150-200 \mathrm{mg} / \mathrm{m}^{2}$ adjuvant temozolomide on days $1-5 / 28$. Three GBM patients relapsed or progressed $<6$ months after diagnosis. Of these, the site of 1 relapse was distant from the primary tumor, outside the radiation field. The other 2 patients did not respond to therapy. These 3 patients were not considered to have had pseudo-progression.

All but one of the GBM patients relapsed during or within the two months following temozolomide chemotherapy. Of these, 11 were rechallenged $<3$ months after the last cycle of chemotherapy. Two others received 1 and 2 cycles of procarbazine and CCNU at first relapse. The other patients had $\geq 3$ months delay until rechallenge with temozolomide.

Anaplastic gliomas. Two males, aged 47 and 58 years with recurrent anaplastic astrocytomas, were pre-treated with concomitant and adjuvant temozolomide. Both progressed with adjuvant temozolomide and received the dose-dense regimen without delay. A 61-year-old female with anaplastic oligodendroglioma was initially pre-treated with PCV and 6 cycles of $200 \mathrm{mg} / \mathrm{m}^{2}$ temozolomide on days $1-5 / 28$ at first relapse. After 17 months, she had a second recurrence and received dose-dense temozolomide.

Treatment. Our dose-dense schedule consisted of $100 \mathrm{mg} / \mathrm{m}^{2}$ temozolomide on days $1-21 / 28$, rounded to increments of $20 \mathrm{mg}$. Patients routinely received PcP-prophylaxis with Cotrimoxazol. The applied dose was increased by increments of $20 \mathrm{mg} /$ day when the total leukocyte count remained at $>6,000 / \mu 1$ over 2 cycles while thrombocytes remained at $>100,000 / \mu 1$. The dose was also reduced by intervals of $20 \mathrm{mg} /$ day when total leukocytes fell to $<3,000 / \mu 1$ or thrombocytes to $<100,000 / \mu 1$.
Table I. Patient characteristics. ${ }^{a}$

\begin{tabular}{|c|c|c|}
\hline \multirow[t]{2}{*}{ Characteristics } & \multicolumn{2}{|c|}{ Patients } \\
\hline & No. & $(\%)$ \\
\hline \multicolumn{3}{|l|}{ Gender } \\
\hline Male & 11 & $(61)$ \\
\hline Female & 7 & $(39)$ \\
\hline \multicolumn{3}{|l|}{ Age (years) } \\
\hline $31-40$ & 3 & $(17)$ \\
\hline $41-60$ & 9 & $(50)$ \\
\hline$>60$ & 6 & (33) \\
\hline Median age (range) & 54.8 & $(32-73)$ \\
\hline \multicolumn{3}{|l|}{ KPS at relapse } \\
\hline $50+60 \%$ & 10 & $(56)$ \\
\hline $70-100 \%$ & 8 & $(44)$ \\
\hline Median KPS (range) & $60 \%$ & $(50-100)$ \\
\hline \multicolumn{3}{|c|}{ Number of progressions } \\
\hline 1 & 14 & $(78)$ \\
\hline 2 & 4 & $(22)$ \\
\hline \multicolumn{3}{|l|}{ Surgical interventions } \\
\hline 1 & 12 & $(67)$ \\
\hline 2 & 4 & $(22)$ \\
\hline 3 & 2 & (11) \\
\hline Prior irradiation & 18 & $(100)$ \\
\hline Prior temozolomide & 18 & $(100)$ \\
\hline Median time to $1 \mathrm{st}$ & 8.2 months & $(2.4-49.2)$ \\
\hline progression (range) & 32.8 weeks & \\
\hline
\end{tabular}

${ }^{\mathrm{a}}$ Glioblastoma only, n=18. KPS, Karnofsky performance status.

Follow-up and documentation. Patients were examined biweekly at our outpatient department, and blood counts were taken weekly. Routine MRI was performed every 3 months (Somatom Trio, Siemens Medical Solutions, Erlangen, Germany: T2w/FLAIR, DWI, PWI, all transversal, 6-mm slice thickness; T2w coronal, 4-mm slice thickness; T1-3D, 1-mm slice thickness, isotropic, without/with gadolinium i.v.). Radiological response was assessed according to the MacDonald criteria (progressive disease, $>25 \%$ increase; partial response, $>50 \%$ reduction of lesions; complete remission, no detectable contrast-enhancing lesion). A deterioration of KPS of $\geq 20 \%$ for more than 4 weeks was considered progressive disease.

\section{Results}

In total, 21 eligible patients (18 GBM, 3 anaplastic glioma) started treatment between May 2005 and April 2007. Of these, 4 patients (2 GBM, 2 anaplastic glioma) are still living as of June 2008, with a median follow-up of 31 months.

Of the 18 GBM patients, 14 were treated at first and 4 at second relapse. Before rechallenge chemotherapy, 4 patients 


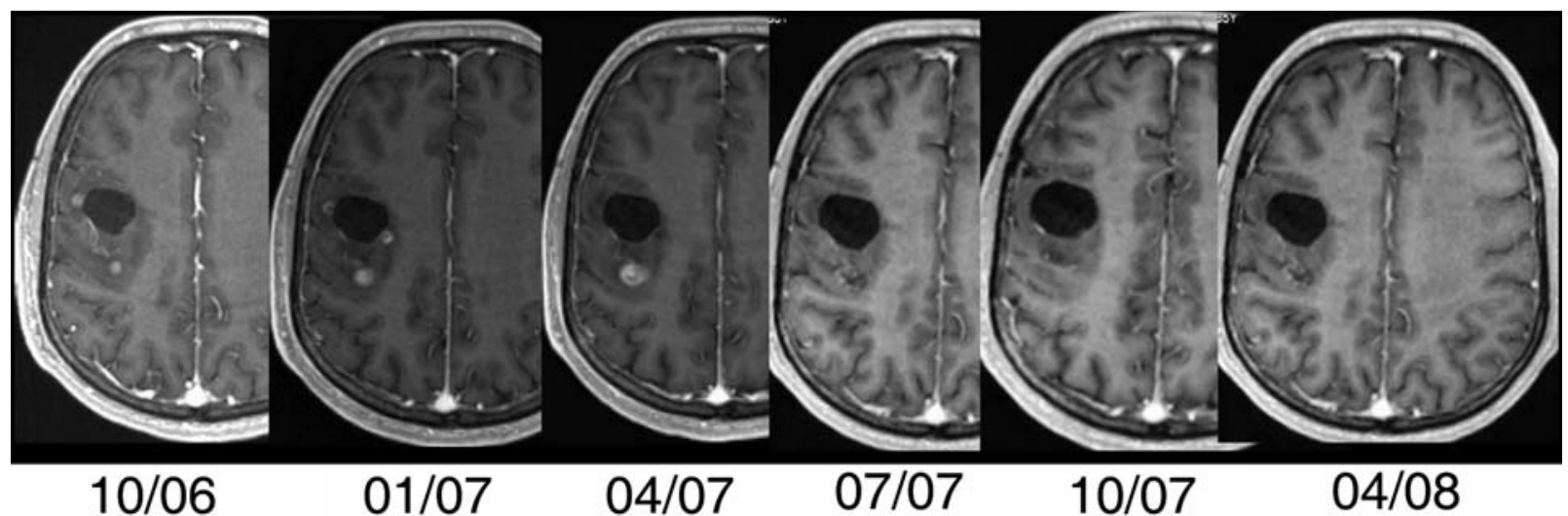

Figure 1. Complete remission in a 34-year-old male diagnosed with glioblastoma in 1/2006 who was pre-treated with focal irradiation and concomitant and adjuvant temozolomide. Beginning in 11/2006, multifocal recurrence was treated with $100 \mathrm{mg} / \mathrm{m}^{2}$ temozolomide on days $1-21 / 28$. The regimen was changed in $4 / 2007$ to $90 \mathrm{mg} / \mathrm{m}^{2}$ on days $1-5 / 7$ because of critical blood counts. During therapy, smaller tumors disappeared first, whereas the larger manifestation was initially slightly progressive and vanished only after 9 months of therapy (axial MRI, T1 + gadolinium). The patient was free of recurrence at MRI control in 7/2008.

Table II. Toxicity. ${ }^{\text {a }}$

\begin{tabular}{lcc}
\hline Toxicity & $\begin{array}{c}\text { Grade } 3 \\
\text { No. }\end{array}$ & $\begin{array}{c}\text { Grade } 4 \\
\text { No. }\end{array}$ \\
\hline Hematotoxicity (total) & 2 & 1 \\
Anemia & 0 & 0 \\
Leukopenia & 3 & 0 \\
Lymphopenia & & \\
Granulozytopenia & 0 & 0 \\
Thrombopenia & 0 & 1 \\
Non-hematological (total) & 1 & 2 \\
Infection (pneumonia after therapy) & 0 & 1 \\
Nausea & 0 & 0 \\
Vomiting & 0 & 0 \\
Fatigue & 0 & 0 \\
Gastrointestinal & 1 & 1 \\
Dose reductions & $11 / 21$ & $48 \%$ \\
Dose increases & $3 / 21$ & $14 \%$ \\
\hline
\end{tabular}

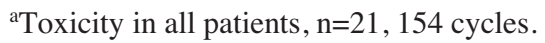

received a second debulking surgery, and 2 patients were operated on a third time. One patient received local BCNU at first recurrence. No patients were re-irradiated. A total of 154 cycles of dose-dense temozolomide was applied to all 21 patients (median 5 cycles, range 2-18). Treatment was discontinued permanently in 2 GBM and 1 anaplastic glioma patient because of long-lasting ( $\geq 3$ months) objective remissions.

Toxicity. Generally, patients tolerated the dose-dense regimen better than the conventional 5/28-day schedule with respect to nausea and fatigue. No treatment-related deaths occurred. An overview of toxicity is provided in Table II.

Hematological toxicity was mild, manageable and reached grade 3 in 3 patients. One patient, however, developed asymptomatic grade 4 thrombopenia and grade 3 leukopenia
Table III. Response to treatment. ${ }^{\mathrm{a}}$

\begin{tabular}{lcc}
\hline Response and survival data & No. & $(\%)$ \\
\hline Best response $\geq 3$ months & & \\
CR & 3 & $(17)$ \\
PR & 1 & $(5)$ \\
SD & 7 & $(39)$ \\
PD & 7 & $(39)$ \\
Objective responses (CR+PR) & 4 & $(22)$ \\
PFS 6-M & 7 & $(39)$ \\
Median survival after relapse & $9.1+$ months \\
Median overall survival & $17.9+$ months \\
\hline
\end{tabular}

${ }^{\mathrm{a}}$ Glioblastoma only, $\mathrm{n}=18$. CR, complete remission; PR, partial response; $\mathrm{SD}$, stable disease; $\mathrm{PD}$, progressive disease. PSF 6-M, 6-month progression-free survival.

during the second course of treatment, requiring transfusions of thrombocytes. After 8 weeks, chemotherapy was restarted at $50 \mathrm{mg} / \mathrm{m}^{2}$ on days $1-5 / 7$ without incident, and the patient was progression-free for 15 months.

One patient developed grade 4 pneumonia several weeks after the termination of chemotherapy for progression. Pneumocystis carinii pneumonia was not isolated.

No immediate severe gastrointestinal toxicity was noted. One severe (grade 3 ) case of constipation was reported, which required discontinuation of therapy for several weeks. This adverse event is considered to be at least in part related to antiemetic co-medication with 5-HT antagonists. One other patient developed a severe (grade 4) increase in liver enzymes. However, the initial elevation of enzymes was documented to have occurred before chemotherapy, when pantoprazole or steroids were the only medications being administered.

Response and survival. Objective responses to therapy were observed not earlier than 3-6 months after treatment. We 
therefore reported response data as 'best response' after at least 3 months (Table III).

Glioblastoma. Of the 18 patients, 3 had a complete and 1 a partial response. Seven patients had stable disease for at least 3 months, while 7 progressed radiologically or clinically within 3 months. Seven patients (39\%) were progression-free after 6 months, 4 after 12 months (22\%) and 1 after 24 months $(6 \%)$. Median overall survival was 17.9 months and median survival from relapse, 9.1 months. The methylation status of the MGMT promoter was determined in 13 patients; 2/7 patients with unmethylated and $3 / 6$ patients with methylated MGMT promoter were progression-free after 6 months . Remission lasted 15 and 19 months in the two patients with unmethylated MGMT promoter.

Anaplastic glioma. All patients with anaplastic gliomas were progression-free after 6 months. One patient with anaplastic astrocytoma is in complete remission after 29 months, and the other is in stable condition 14 months after relapse.

\section{Discussion}

While temozolomide-based chemotherapy has been established as a primary therapy of GBMs, no standard regimen exists for the treatment of tumor recurrence. Here, we provide evidence that $100 \mathrm{mg} / \mathrm{m}^{2}$ temozolomide on days $1-21$ of 28 is active in recurrent malignant gliomas even after pre-treatment with temozolomide in patients with unfavorable prognostic indicators.

MGMT was recently identified as a major predictor of response to chemotherapy that can be influenced by therapy. This has focused attention on alternative therapeutic regimens with prolonged application of increased doses of temozolomide per 28-day cycle to overcome resistance to the conventional regimen. For this approach, either the application of $150 \mathrm{mg} / \mathrm{m}^{2}$ on days $1-7 / 14\left(2100 \mathrm{mg} / \mathrm{m}^{2}\right.$ per cycle) (10) or $75 \mathrm{mg} / \mathrm{m}^{2}$ on days $1-21 / 28\left(1575 \mathrm{mg} / \mathrm{m}^{2}\right.$ per cycle) (8) has been used. Data from peripheral blood mononuclear cells during therapy indicate that sustained depletion of MGMT for at least 3 weeks can be achieved when temozolomide is administered over 21 days, while it regains normal values every second week with administration on days 1-7/14 (7). We therefore decided to use the 21/28-day regimen, but increased the daily dose for two reasons: i) previous results indicated that the $7 / 14$ regimen, which added up to a dose of $2100 \mathrm{mg} / \mathrm{m}^{2}$ per cycle, was associated with an acceptable toxicity, while the 21 day schedule with $75 \mathrm{mg} / \mathrm{m}^{2}$ only added up to $1575 \mathrm{mg} / \mathrm{m}^{2}$ per cycle; ii) in vitro studies indicate that temozolomide can induce apoptosis in GBM cells in a dose-dependent manner requiring the equivalent of at least $100 \mathrm{mg} / \mathrm{m}^{2}$ (11). This dosage has been shown to be tolerable in patients with renal cell carcinoma (12), and has shown promising results in patients with recurrent anaplastic gliomas (13).

Toxicity. The data in our series demonstrate that the chosen regimen is feasible and its toxicity manageable. The lower rate of nausea and fatigue can be explained by the lower daily dose of temozolomide. In fact, one patient restarted work under this regimen. In general, blood counts decreased continuously, allowing for a gradual dose adaptation that we found appropriate to perform by increments of $20 \mathrm{mg}$ of absolute dose. In this manner, dosage was increased in selected patients. An uncontrolled drop in blood counts was experienced in only 1 of the 21 patients. This hematotoxicity was managed without hospital admission, and the patient was progression-free for 15 months after first relapse. In this case and in other patients with critical blood counts, the application mode was switched to a 5/7-day regimen. This resulted in even more homogeneous leukocyte and thrombocyte counts than under the 21/28-day regimen. We therefore do not consider hematotoxicity to be a major obstacle to this schedule.

Major concerns have recently emerged regarding an increase in infections under the 21/28-day regimen, secondary to selective lymphopenia. Tosoni et al reported a considerable rate of pneumonia during a 21/28-day regimen for relapsed malignant glioma (14). However, a similar rate of infection was not observed by Neyns et al (13), who treated patients using the same regimen. In our series, 1 patient developed severe pneumonia 3 weeks after permanent discontinuation of chemotherapy for progression, without isolation of Pneumocystis carinii. PcP-prophylaxis, the administration of the lowest possible dose of steroids, and close clinical guidance may have been responsible for the low rate of infections observed.

Unexpectedly, we experienced two major gastrointestinal events. One case of severe constipation requiring temporary interruption of chemotherapy was probably primarily due to almost continuous antiemetic therapy with $5-\mathrm{HT}_{3}$ antagonists. We switched to Alizapride in most of the patients, and observed lower rates of constipation. Only approximately $25 \%$ of all patients still needed 5- $\mathrm{HT}_{3}$ antagonists, and these were closely observed for gastrointestinal side effects. One other patient developed a grade 4 elevation of liver enzymes, which had however started prior to chemotherapy, during treatment with steroids and pantoprazol. We therefore do not consider temozolomide to be the cause of this toxicity.

Efficacy. In the literature, response to treatment with chemotherapy alone ranges from 3.5 to $21 \%$ with a nitrosourea-based regimen $(4,15)$, and from $5.4 \%$ with conventionally-dosed temozolomide (16) to $9.5 \%$ with dose-dense temozolomide (10). PFS-6M was 32-38\% and median survival from relapse was approximately 35 weeks. In experimental studies with targeted therapies, PFS-6M ranged from 30 to $40 \%$ and median survival from relapse between 20 and 40 weeks (16-18). In these series, the median KPS was $\geq 80 \%$. Patients with KPS of $50 \%$ were almost always not included. Although the clinical prognostic indicators in our series were less favorable, the objective response rate of $22 \%$ is encouraging. Pseudo-progression, due to changes in contrast-enhancing volume early after radiotherapy, was obviously not a reason for this positive result, since 2 of 3 patients with recurrences within 6 months had subsequent progressive disease, while the site of the third relapse was distant from the primary tumor location. The PFS-6M of 39\% and median survival after relapse of 39 weeks in our GBM patients compare well with the most successful studies on recurring GBMs. Notably, long-term responses of 15 and 19 months were even achieved in patients with unmethylated MGMT promoter, pointing towards the efficacy of this MGMT-depleting strategy. The 
long-term response of all patients with grade III tumors indicates that the regimen is also effective in patients with anaplastic gliomas, an observation that supports recentlypublished promising results (13). Also of note is that objective responses and a favorable long-term outcome were observed even though, in a considerable number of patients, the regimen was switched, directly or within less than 3 months, from the conventional 5/28-day to the dose-dense 21/28-day regimen. Pausing temozolomide administration before rechallenge therefore appears unnecessary, and may result in valuable time being lost. Response to treatment was not achieved before a delay of at least 3 months, during which time salvage therapy did not succeed in preventing neurological deterioration.

In conclusion, we demonstrated that rechallenge with temozolomide is effective in recurrent malignant gliomas when administered under a dose-dense regimen. Toxicity is acceptable and manageable, although gastrointestinal toxicity and grade 4 hematotoxicity indicate the need for close patient observation during therapy. In our view, an initial daily dose of $100 \mathrm{mg} / \mathrm{m}^{2}$ in a prolonged schedule with subsequent individual dose adaptation is the best compromise between toxicity and efficacy. Switching to a 1-5/5-day regimen allowed for even better dose adaptation in 6 patients with critical blood counts; in some of these a dose of $100 \mathrm{mg} / \mathrm{m}^{2}$ was maintained. Previous studies indicate that MGMT requires approximately 1 week to regain normal levels and will not recover within a 2-day break of temozolomide administration. MGMT is thus expected to be permanently depleted under the 5/7 regimen, and the efficacy of treatment may be optimized. Therefore, we now initially treat patients with recurrent gliomas with a 5/7-day 'Temozolomide-glioma-working day-dose-dense' schedule (TEGWONDO). Toxicity and efficacy will be evaluated prospectively.

\section{Acknowledgements}

We gratefully acknowledge G. Nagel for technical assistance. This study was supported by DFG-KA724.

\section{References}

1. Weller M, Muller B, Koch R, Bamberg M and Krauseneck P: Neuro-Oncology Working Group 01 trial of nimustine plus teniposide versus nimustine plus cytarabine chemotherapy in addition to involved-field radiotherapy in the first-line treatment of malignant glioma. J Clin Oncol 21: 3276-3284, 2003.

2. Stupp R, Mason WP, van den Bent MJ, Weller M, Fisher B, Taphoorn MJ, Belanger K, Brandes AA, Marosi C, Bogdahn U, Curschmann J, Janzer RC, Ludwin SK, Gorlia T, Allgeier A, Lacombe D, Cairncross JG, Eisenhauer E and Mirimanoff RO: Radiotherapy plus concomitant and adjuvant temozolomide for glioblastoma. N Engl J Med 352: 987-996, 2005.

3. Wong ET, Hess KR, Gleason MJ, Jaeckle KA, Kyritsis AP, Prados MD, Levin VA and Yung WK: Outcomes and prognostic factors in recurrent glioma patients enrolled in phase II clinical trials. J Clin Oncol 17: 2572-2578, 1999.
4. Schmidt F, Fischer J, Herrlinger U, Dietz K, Dichgans J and Weller M: PCV chemotherapy for recurrent glioblastoma. Neurology 66: 587-589, 2006.

5. Yung WK, Albright RE, Olson J, Fredericks R, Fink K, Prados MD, Brada M, Spence A, Hohl RJ, Shapiro W, Glantz M, Greenberg H, Selker RG, Vick NA, Rampling R, Friedman H, Phillips P, Bruner J, Yue N, Osoba D, Zaknoen S and Levin VA: A phase II study of temozolomide vs. procarbazine in patients with glioblastoma multiforme at first relapse. Br J Cancer 83: 588-593, 2000.

6. Hegi ME, Diserens AC, Gorlia T, Hamou MF, De Tribolet N, Weller M, Kros JM, Hainfellner JA, Mason W, Mariani L, Bromberg JE, Hau P, Mirimanoff RO, Cairncross JG, Janzer RC and Stupp R: MGMT gene silencing and benefit from temozolomide in glioblastoma. N Engl J Med 352: 997-1003, 2005.

7. Tolcher AW, Gerson SL, Denis L, Geyer C, Hammond LA, Patnaik A, Goetz AD, Schwartz G, Edwards T, Reyderman L, Statkevich P, Cutler DL and Rowinsky EK: Marked inactivation of $\mathrm{O}^{6}$-alkylguanine-DNA alkyltransferase activity with protracted temozolomide schedules. Br J Cancer 88: 1004-1011, 2003.

8. Brandes AA, Tosoni A, Cavallo G, Bertorelle R, Gioia V, Franceschi E, Biscuola M, Blatt V, Crino L and Ermani M: Temozolomide 3 weeks on and 1 week off as first-line therapy for recurrent glioblastoma: phase II study from Gruppo Italiano Cooperativo di Neuro-oncologia (GICNO). Br J Cancer 95: 1155-1160, 2006.

9. MacDonald DR, Cascino TL, Schold SC Jr and Cairncross JG: Response criteria for phase II studies of supratentorial malignant glioma. J Clin Oncol 8: 1277-1280, 1990.

10. Wick A, Felsberg J, Steinbach JP, Herrlinger U, Platten M, Blaschke B, Meyermann R, Reifenberger G, Weller M and Wick W: Efficacy and tolerability of temozolomide in an alternating weekly regimen in patients with recurrent glioma. J Clin Oncol 25: 3357-3361, 2007.

11. Roos WP, Batista LF, Naumann SC, Wick W, Weller M, Menck CF and Kaina B: Apoptosis in malignant glioma cells triggered by the temozolomide-induced DNA lesion $\mathrm{O}^{6}$-methylguanine. Oncogene 26: 186-197, 2007.

12. Bex A, Kerst J, Mallo H, van Tinteren H, Horenblas S and De Gast GC: Extended continuous oral temozolomide in patients with progressive metastatic renal cell carcinoma not responding to interferon alpha $2 \mathrm{~b}$. J Chemother 17: 674-678, 2005.

13. Neyns B, Chaskis C, Joosens E, Menten J, D'Hondt L, Branle F, Sadones J and Michotte A: A multicenter cohort study of dosedense temozolomide (21 of 28 days) for the treatment of recurrent anaplastic astrocytoma or oligoastrocytoma. Cancer Invest 26: 269-277, 2008.

14. Tosoni A, Cavallo G, Ermani M, Scopece L, Franceschi E, Ghimenton C, Gardiman M, Pasetto L, Blatt V and Brandes AA: Is protracted low-dose temozolomide feasible in glioma patients? Neurology 66: 427-429, 2006.

15. Brandes AA, Tosoni A, Basso U, Reni M, Valduga F, Monfardini S, Amista P, Nicolardi L, Sotti G and Ermani M: Second-line chemotherapy with irinotecan plus carmustine in glioblastoma recurrent or progressive after first-line temozolomide chemotherapy: a phase II study of the Gruppo Italiano Cooperativo di Neuro-Oncologia (GICNO). J Clin Oncol 22: 4779-4786, 2004.

16. Groves MD, Puduvalli VK, Hess KR, Jaeckle KA, Peterson P, Yung WK and Levin VA: Phase II trial of temozolomide plus the matrix metalloproteinase inhibitor, marimastat, in recurrent and progressive glioblastoma multiforme. J Clin Oncol 20: 1383-1388, 2002.

17. Dresemann G: Imatinib and hydroxyurea in pretreated progressive glioblastoma multiforme: a patient series. Ann Oncol 16: 1702-1708, 2005.

18. Vredenburgh JJ, Desjardins A, Herndon JE, Dowell JM, Reardon DA, Quinn JA, Rich JN, Sathornsumetee S, Gururangan S, Wagner M, Bigner DD, Friedman AH and Friedman HS: Phase II trial of bevacizumab and irinotecan in recurrent malignant glioma. Clin Cancer Res 13: 1253-1259, 2007. 